phys. stat. sol. (b) 221, 133 (2000)

Subject classification: 78.45.+h; 78.66.Fd; 78.90.+t; S7.12

\title{
Patterns in Broad-Area Microcavities
}

\author{
T. Ackemann ${ }^{1}$ ) (a), S. Barland (a), M. Giudici (a), J. R. Tredicce (a), \\ S. BAlle (b), R. JÄGer (c), M. Grabherr (c), M. Miller (c), and K. J. Ebeling (c) \\ (a) Institut Non-Linéaire de Nice, UMR 6618, CNRS-UNSA, 1361 Route des Lucioles, \\ F-06560 Valbonne, France
}

(b) Departamento de Física Interdisciplinar, Instituto Mediterráneo de Estudios Avanzados, IMEDEA (CSIC-UIB), E-07071 Palma de Mallorca, Spain

(c) Department of Optoelectronics, University of Ulm, Albert-Einstein-Allee 45, D-89069 Ulm, Germany

(Received April 10, 2000)

The emergence of spatial structures with an approximate hexagonal symmetry is observed in broad-area amplifying microcavities with injection of a homogeneous beam. The length scale is controllable via detuning. Optical bistability occurs close to the longitudinal resonance.

Semiconductor microcavities display a variety of complex behaviour resulting from the strong coupling between light field and medium, often a semiconductor quantum well or a set of quantum wells. The observed features include the formation and nonlinear optics of polaritonic quasiparticles formed from excitons and photons (see e.g. [1, 2]) as well as spontaneous gain due to collective bosonic phenomena [3]. In active microcavities, i.e. vertical-cavity surface-emitting lasers (VCSEL), squeezing was found [4]. The angular selectivity of the high-quality microcavity in conjunction with the possibility to grow structures with a large extent in the transverse direction implies also the potential for the formation of complex spatial structures, which became first apparent in VCSELs (see e.g. [5, 6]). Recent experiments resulted in the observation of spatial patterns also in driven passive cavities [7, 8]. We report on an intermediate case, in which a broadarea VCSEL is operated as a regenerative amplifier, i.e. biased electrically below threshold but above transparency and subjected to external injection of a homogeneous beam. Recent works predict the spontaneous emergence of hexagonal patterns and the possibility of bistable soliton-like excitations (cavity solitons) in such systems [9, 10], which might enable new forms of all-optical, massively parallel information processing. Partly electrically pumped systems appear to be advantageous in some respects compared to purely passive systems [7,8], since cascading is easier to achieve and the requirements on optical power are considerably lower. First results were presented in [11].

The devices under study are based on three InGaAs/GaAs quantum wells of thickness $8 \mathrm{~nm}$ embedded in a spacer layer with a thickness of one wavelength. The cavity is closed by Bragg reflectors with 30 stacks on the p-side and 20.5 on the n-side. A de-

1) Permanent address: Institut für Angewandte Physik, WWU Münster, Corrensstraße 2/4, D-48149 Munster, Germany; Tel.: +251-83335-53; Fax: -13; e-mail: ackeman@uni-muenster.de 


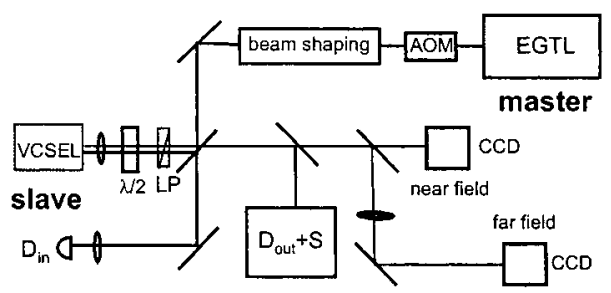

Fig. 1. Scheme of experimental set-up: EGTL external grating tunable laser, AOM acousto-optical modulator, beam shaping optics is an amorphic prism pair and spatial filter, $\lambda / 2$ half-waveplate, LP linear polarizer, CCD charge-coupled device camera, D detector, S spectrometer

tailed description of the devices can be found in [12]. The active area is circular (diameter $\approx 35$ to $55 \mu \mathrm{m}$ ). Its size is defined by a thin oxide aperture. Emission takes place through the thinned, anti-reflection coated substrate on the n-side. Most of the devices under study were operated without prior packaging (on-wafer testing). The free running lasers start at threshold typically in a high order Laguerre-or Hermite Gaussian mode [13].

Figure 1 shows a schematic view of the set-up. The spatially filtered beam of a tunable semiconductor master oscillator is injected in the VCSEL such that it fills the aperture. The maximum available power level in front of the VCSEL amounts to some mWs and is controlled by an acousto-optical modulator. The injected beam is linearly polarized. Coupling to the VCSEL is achieved by an aspheric lens. The near field (NF, i.e. the plane of the active zone) and far field (FF, the Fourier spectrum) intensity distributions are imaged onto two charge-coupled device (CCD) cameras. Output and injected power and the spectral characteristics are monitored also.

The spatially integrated small-signal gain can be very high $(\gtrsim 50$ in resonance) in accordance with earlier studies [14]. The amplification curve is rather narrow $(<0.3 \mathrm{~nm}$ HWHM) but there is a slowly decaying low-amplitude tail for blue detuning of the master oscillator with respect to the cavity resonance, which is due to the fact that resonances belonging to spatial degrees of freedom are excited. Typical emerging structures are shown in Fig. 2. The slave was biased at 97\% of threshold but the results are not sensitive to this particular value. The near field patterns (Fig. 2a, c, e) display a modulated symmetry-broken structure. Beside spontaneous emission the Fabry-Perot spectrum displays only one peak at the injection wavelength (HWHM $3 \mathrm{GHz}$, resolution limited) indicating that the emission is coherent over the whole aperture. The far field intensity distributions (Fig. 2b, d) show distinct contributions on a ring in Fourier space. The radius of the ring reduces if the detuning is decreased. At the same time the
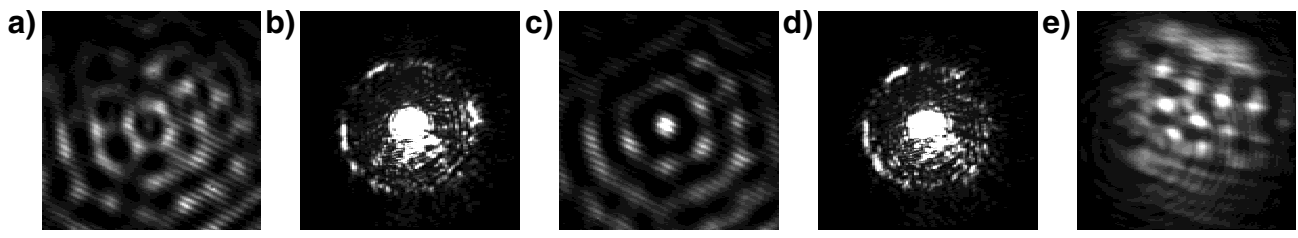

Fig. 2. Typical spatial structures observed for blue detuning (in a), b) $1.3 \mathrm{~nm}$; c), d) $1.1 \mathrm{~nm}$, e) $1.2 \mathrm{~nm}$ ) of the master oscillator with respect to the longitudinal cavity resonance. a), c), e) display the near field; b), d) the the corresponding far field images of a), c). The device in a), c) is a $54 \mu \mathrm{m}$ diam. VCSEL operated on-wafer with a probe tip, the device in e) is mounted on a diamond heat spreader and wire bonded (see [12], diam. $38 \mu \mathrm{m}$ ). The far-field images are overexposed at the dc peak, which corresponds to the amplified injected field itself, in order to visualize the off-axis emission. Also the peaks in e) are overexposed 
near field patterns coarsen, of course. A quantitative analysis shows that the square of the wavevector depends linearly on detuning (Fig. 3). Such a scaling is expected for diffractive patterns in a plano-planar cavity $[15,16,9,10]$. The tilt of the waves with respect to the optical axis reestablishes the resonance condition in the cavity. Therefore the off-axis waves are spontaneously generated from the injected on-axis wave which is not in resonance. Using $n_{0}=3.5$ as an average refractive index for the semiconductor material one can reproduce not only the scaling but also the absolute value of the resonant wavevector from the usual Fabry-Perot theory [11] (solid line in Fig. 3, the intercept with $q=0$ is fitted since the position of the longitudinal resonance is not known a priori).

The modulation in the near field patterns is visible in a detuning range of about 3.5 $\mathrm{nm}$. The modulation depth increases with decreasing detuning [11]. There is a tendency that the modulations arrange along rings (Fig. 2a, c). The inner part of these patterns displays an approximate sixfold rotational symmetry either in the form of bright spots (Fig. 2c) or dark holes (Fig. 2a). This observation verifies the prediction of hexagons [9], though there is apparently still some influence from the circular boundary conditions. Such a competition between intrinsic dynamical and boundary induced symmetry is known in several other optical (see e.g. [17]) and non-optical (see e.g. [18]) pattern forming systems. In a device mounted on a head spreader there was a small range of parameters in which the underlying rings disappear and true symmetry breaking takes place (Fig. 2e). This might be due to the fact that axisymmetric radial inhomogeneities due to thermal lensing are less important in this device, but this point needs further investigation.

The observed patterns are different from the patterns in free running broad-area lasers $[15,5,13]$ since the external field breaks the phase invariance and also the inversion symmetry of the underlying equations. Therefore, amplitude equations for the nonlinear evolution contain quadratic terms (see e.g. [16] for purely passive driven cavities) in contrast to the normal third-order Lamb laser theory. Quadratic terms are known to favor hexagons [19]. Due to the breaking of the inversion symmetry there are two different kinds of patterns: negative hexagons, which consist of dark holes on a back-
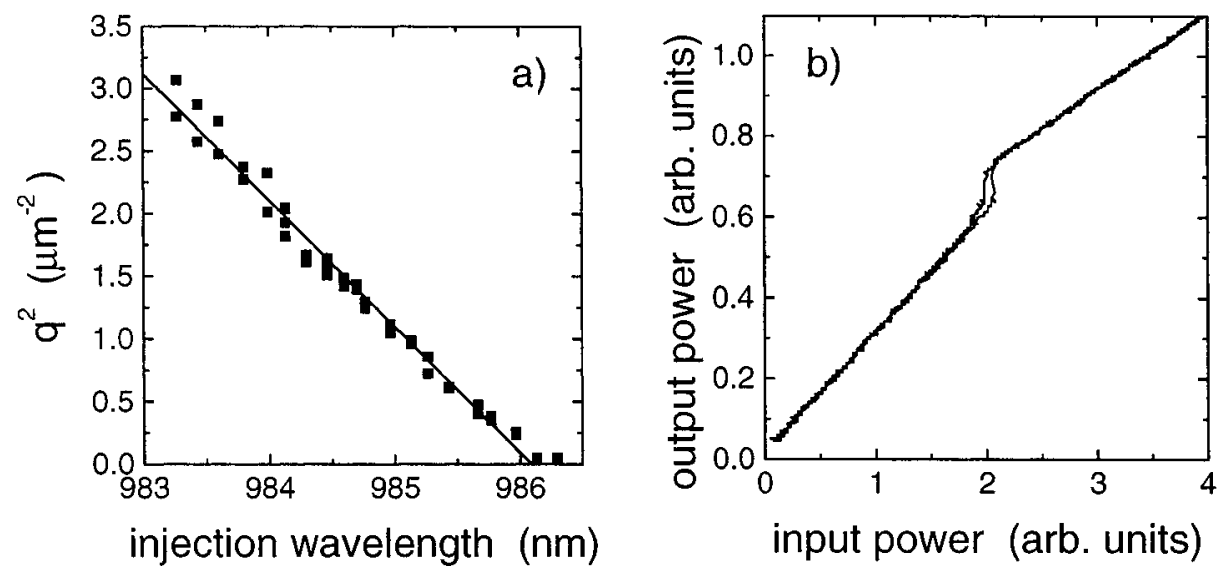

Fig. 3. a) Square of transverse wavevector in dependence on the injection wavelength. b) Output power versus injected power close to the longitudinal resonance, $\lambda \approx 986.1 \mathrm{~nm}$ 
ground intensity (Fig. 2a), and positive hexagons, which consist of bright peaks on a background intensity (Fig. 2c).

Close to the longitudinal resonance optical bistability, i.e. the coexistence of a high transmission state and a low transmission state, is observed in a very narrow range of detuning. Hysteretic switching takes place if the input power is ramped up and down slowly (Fig. 3b). The bistable states correspond to two different amplitudes of a small filamentary peak in the laser aperture. At the longitudinal resonance an ideal device should display homogeneous emission, in the devices under study we observe the transition to a filament if the length scale of the pattern (Fig. 3a) becomes of the order of the laser aperture. The emergence of the filament and its position is apparently due to inhomogeneities. The bistability is very sensitive to fluctuations and the parameter window with optical bistability is considerably narrower than predicted in [10]. This effect and its possible connection to gradient inhomogeneities in the cavity resonance [20] has to be investigated in more detail.

Acknowledgements T. A. gratefully acknowledges financial support from the Alexander von Humboldt-Stiftung. The work was supported by the PIANOS project of the European Union (project No. 28235).

\section{References}

[1] C. Weisbuch, M. Nishioka, A. Ishikawa, and Y. Arakawa, Phys. Rev. Lett. 69, 3314 (1992).

[2] F. JAHNKE et al., Phys. Rev. Lett. 77, 5257 (1996).

[3] P. G. SAvvidis et al., Phys. Rev. Lett. 84, 1547 (2000).

[4] D. Wiedenmann et al., Appl. Phys. Lett. 75, 3075 (1999).

[5] S. Hegarty, G. Huyet, J. G. McInerney, and K. D. Choquette, Phys. Rev. Lett. 82, 1434 (1999).

[6] J. SCheuer and M. ORENSTEIN, Science 285, 230 (1999).

[7] V. B. Taranenko, I. Ganne, R. J. Kuszelewicz, and C. O. Weiss, Phys. Rev. A 61, 063818 (2000).

[8] G. Slekys, I. Ganne, I. Sagnes, and R. Kuszelewicz, J. Opt. B 2, 443 (2000).

[9] M. Brambilla et al., Phys. Rev. Lett. 79, 2042 (1997).

[10] L. Spinelui et al., Phys. Rev. A 58, 2542 (1998).

[11] T. ACKemann et al., Opt. Lett. 25, 814 (2000).

[12] M. GrabherR et al., IEEE Photon. Tech. Lett. 10, 1061 (1998).

[13] T. ACKEMANn et al., J. Opt. B 2, 406 (2000).

[14] D. Wiedenmann, B. Moeller, R. MichalziK, and K. J. Ebeling, Electronics Lett. 32, 342 (1996).

[15] P. Jakobsen, J. Moloney, A. Newell, and R. Indik, Phys. Rev. A 45, 8129 (1992).

[16] W. J. FirTh and A. J. SCROGgIE, Europhys. Lett. 26, 521 (1994).

[17] R. Neubecker, B. Thuering, and T. Tschudi, Chaos, Solitons \&tab; Fractals 4, 1307 (1994).

[18] C. W. Meyer, D. S. Cannel, and G. Ahlers, Phys. Rev. A 45, 8383 (1992).

[19] F. H. Busse, Rep. Progr. Phys. 41, 1929 (1978).

[20] S. Coen, M. Tlidi, P. Emplit, and M. Haelterman, Phys. Rev. Lett. 83, 2328 (1999). 